\title{
Sizing the aortic annulus with a robotised, commercially available soft balloon catheter: in vitro study on idealised phantoms
}

\author{
Andrea Palombi ${ }^{1}$, Giorgia M Bosi ${ }^{1}$, Sara Di Giuseppe ${ }^{2}$, Elena De Momi ${ }^{2}$, \\ Shervanthi Homer-Vanniasinkam ${ }^{1}$, Gaetano Burriesci ${ }^{1,3}$, Helge A Wurdemann ${ }^{1}$
}

\begin{abstract}
Transcatheter aortic valve implantation (TAVI) is a minimally invasive surgical technique to treat aortic heart valve diseases. According to current clinical guidelines, the implanted prosthetic valve replacing the native one is selected based on pre-operative size assessment of the aortic annulus through different imaging techniques. That very often leads to suboptimal device selection resulting in major complications, such as prosthetic valve leakage or interruption of the cardiac electrical signal.

In this paper, we propose a new, intra-operative approach to determine the diameter of theaortic annulus exploiting intraballoon pressure and volume data, acquired from a robotised valvuloplasty balloon catheter. An inflation device, capable of collecting real-time intra-balloon pressure and volume data, was designed and interfaced with a commercially available valvuloplasty balloon catheter. A sizing algorithm allowing to precisely estimate the annular diameter was integrated. The algorithm relies on a characterised analytical model of the balloon free inflation and an iterative method based on linear regression.

In vitro tests were performed on idealised aortic phantoms. Experimental results show that pressure-volume data can be used to determine annular diameters bigger than the unstretched diameter of the balloon catheter. For these cases, the proposed approach exhibited good precision (maximum average error $0.93 \%$ ) and good repeatability (maximum standard deviation $\pm 0.11 \mathrm{~mm})$.
\end{abstract}

\section{INTRODUCTION}

Aortic stenosis (AS) is a serious heart valve disease, which affects $2 \%$ of people who are over 65 years of age [1]. The condition is characterised by a narrowing of the aortic valve opening, usually induced by age-related progressive calcifications. If untreated, AS is associated with a $50 \%$ rate of death within two years after the appearance of symptoms [2]. Open-heart surgical aortic valve replacement (AVR) is the traditional procedure used to treat AS. However, at least $30 \%$ of the patients with severe AS cannot undergo surgery due to advanced age, left ventricular dysfunction or multiple coexisting health conditions [1], [2]. Hence, transcatheter aortic valve implantation (TAVI) was introduced in 2002 as a minimally invasive alternative to AVR. During TAVI, the native valve is first dilated using a balloon catheter, designed to reach a specific diameter for a given pressure. The balloon size is usually chosen according to imaging-based assessment of the annular diameter [3]-[5]. This procedural step is called

\footnotetext{
${ }^{1}$ Department of Mechanical Engineering, University College London, London WC1E 7JE, UK (corresponding author e-mail: h.wurdemann@ucl.ac.uk).

${ }^{2}$ Electronic Information and Bioengineering Department, Politecnico di Milano, 20133 Milano, Italy.

${ }^{3}$ Bioengineering Group at Ri.MED Foundation, 90133 Palermo, Italy.
}

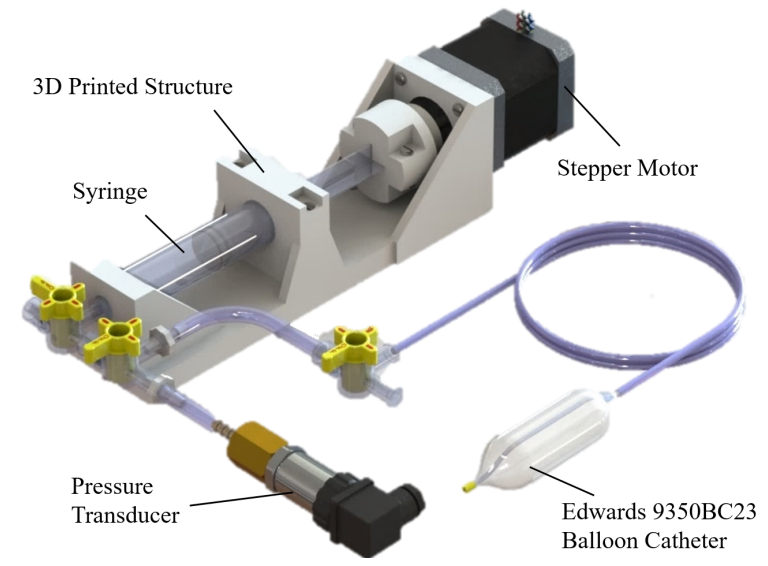

Fig. 1. Robotic valvuloplasty balloon catheter: the commercially available valvuloplasty balloon catheter is interfaced with a motorised inflation device and a pressure transducer.

balloon aortic valvuloplasty (BAV). A different catheter is then utilised to implant a bio-prosthetic device within the native diseased valve [6], [7]. The positive clinical outcomes have allowed this technique to rapidly develop over the past fifteen years. Improved prostheses and delivery systems as well as the increased experience of surgeons favoured the use of TAVI even in patients who are at low or intermediate surgical risk [8], [9].

Nevertheless, TAVI is still affected by some major intra-operative complications, such as prosthetic valve leakages (called aortic regurgitation) and interruption of the cardiac electrical signal (called atrioventricular blocks) [9]-[13] Residual mild leaks associated with higher mortalities at two years are reported in up to $61 \%$ of patients after TAVI [13], [14]. The main causes of regurgitation are malpositioning or undersizing of the prosthetic device [10]-[12], an elliptic shape of the aortic annulus or an irregular distribution of calcium depositions within the surrounding tissue [15], [16]. In such cases, the expansion of the prosthetic valve might be incomplete or uneven. On the other hand, an oversized prosthesis is a predictor of future requirement of a permanent pacemaker [9]. Hence, choosing the right valve size is crucial to prevent any of these complications.

According to current clinical guidelines, the prosthetic valve selection is essentially based on pre-operative size assessment of the aortic annulus. This is usually performed either with 2D transesophageal echocardiography (TEE) or transthoracic echocardiography (TTE) [17]. However, it has 
been demonstrated that bidimensional echocardiographic images are not the best option to assess the complex 3D geometry and the elliptic shape of the aortic annulus, since they lead to underestimation of the annular diameter [17][20]. Multidetector computer tomography (MDCT) has been used to accurately reconstruct a 3D model of the aortic root [17], [18]. Nevertheless, MDCT has some procedural limitations. In particular, the risk of contrast nephropathy is very high in patients with renal morbidity, which is quite common in elderly people affected by AS [19], [20]. More recently, 3D transesophageal echocardiography (3D-TEE), which offers precision comparable to MDCT and improved safety, has been proposed as the first image modality of choice to preoperatively assess the annular dimensions [20]. Still, such technology is not routinely available [21].

In addition to the described modality-specific drawbacks, the current approach for prosthetic valve selection relies on preoperative geometrical data only. Therefore, potential changes in the annular geometry are neglected though widening of the annulus has been hypothesised to directly impact the annular geometry in up to $25 \%$ of the patients [22]. To overcome the aforementioned problems, the idea of using a BAV balloon catheter, as a support for prosthetic valve selection, has been proposed in the literature. Different methods based on standard aortic balloons and manual inflation devices have been outlined [17], [23]-[25]. Although these can be used to confirm or refute the size of the pre-operatively selected prosthesis, they cannot provide an actual measurement of the annular diameter. Hence, the support they offer for device selection is limited.

In this paper, we propose a new, intra-operative approach to determine the diameter of the aortic annulus exploiting intra-balloon pressure and volume data, acquired from a robotic valvuloplasty balloon catheter (Figure 1). An inflation device capable of collecting real-time intra-balloon pressure and volume data was designed and interfaced with a commercially available BAV balloon catheter. A sizing algorithm allowing to estimate the annular diameter from obtained pressure-volume data was also implemented. The performance of the proposed approach was assessed through experimental tests on idealised rigid aortic phantoms.

Section II describes the overall robotic platform, the analytical balloon model and the sizing algorithm. The experimental setup with idealised aortic phantoms and the test protocol as well as experimental results are discussed in Section III. In Section IV, the achievements of this work are summarised and future work is outlined.

\section{System Design And Methodology}

This section describes the interface of a commercially available BAV balloon catheter with a motorised inflation device and pressure transducer to monitor intra-balloon pressure and volume data during in vitro valvuloplasty. Further, this information is fed into an algorithm based on the analytical model of the balloon catheter to determine the diameter of an idealised annulus.

\section{A. The robotic inflation device}

As shown in Figure 1, the inflation device consists of a $10 \mathrm{ml}$ syringe (Terumo Corporation, Shibuya, Tokyo, Japan), fixed on a 3D printed structure made of VisiJet EX200 resin (3D Systems, Rock Hill, SC, US). The movement of the plunger is regulated by a Nema 17 non-captive linear stepper motor with anti-rotation mechanism (17HS5001-100D8, RobotDigg, Shanghai, China). Considering the geometry of the syringe and the specifications of the stepper motor, an amount of $0.008 \mathrm{ml}$ fluid is approximately delivered into the balloon by a single step. Hence, the intra-balloon volume can constantly be monitored by simply counting the number of executed steps. Pressure data is acquired using a PXM319-007A10V absolute pressure transducer (Omega Engineering Inc., Stamford, CT, US). The motor and the sensor are interfaced with an Arduino Uno microcontroller combined with a VMA03 motor shield (Velleman, Gavere, Belgium) (Figure 2). The shield allows to exploit an external power supply and four digital pins of the Arduino board to control motor direction and speed. A single analog input of the microcontroller is used to acquire pressure data. The balloon catheter employed in this paper operates over an absolute pressure range of $0-5 \mathrm{~atm}(\simeq 0-0.5 \mathrm{MPa})$. Since the maximum analog input voltage of the Arduino is $5 \mathrm{~V}$, a voltage divider is required to rescale the output of the transducer $(0-7$ bar $=0.7 \mathrm{MPa} ; 0-10 \mathrm{~V})$ and make our pressure range of interest readable (Figure 2).

A system of PVC pipes and 4-way stopcocks with luer lock fittings (Cole-Parmer, Vernon Hills, IL, US) is used to hydraulically connect the balloon catheter and the sensor to the syringe (Figure 1).

The robotic inflation device is entirely managed by a program developed in Processing, an open-source Java-based programming language and integrated development environment. The program relies on the Firmata protocol, which is implemented in the firmware uploaded on the Arduino, to communicate with the microcontroller via USB cable.

The modality of operation (Inflation/Deflation) and the amount of fluid to be introduced/removed are specified

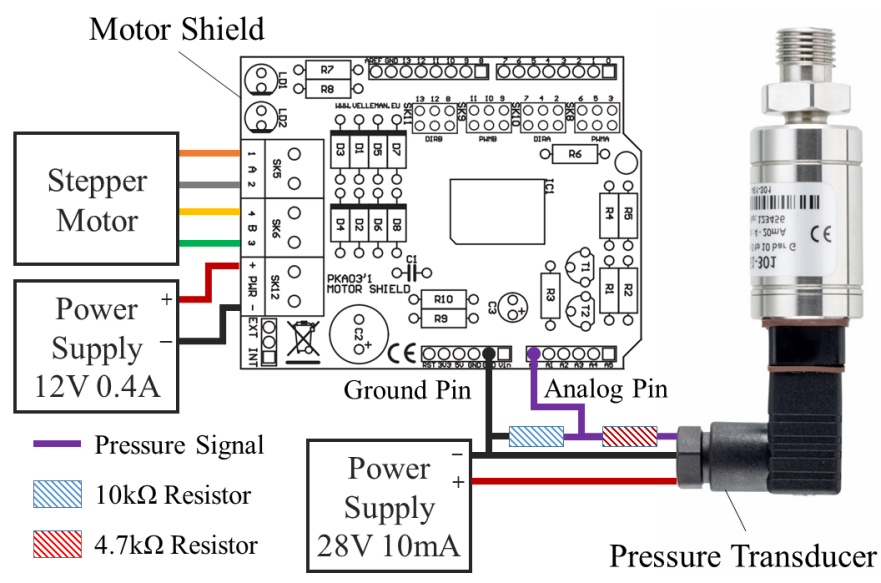

Fig. 2. An Arduino Uno board with a motor shield stacked on top is used to both control the stepper motor and acquire pressure data. 
through a graphical user interface (GUI). Inputs from the GUI are fed to an automated routine, which drives the stepper and regulates data acquisition as shown in Algorithm 1. At the end of the routine, a text file, containing an absolute pressure value in [atm] and a volume value in [ml] for each step executed by the motor is exported.

\section{B. Balloon analytical model for diameter estimation}

The integrated balloon catheter is an Edwards 9350BC23 (Edwards Lifesciences, Irvine, CA, US). The device is designed to reach a diameter of $23 \mathrm{~mm}$ at the nominal intraballoon gauge pressure of $4 \mathrm{~atm}(\simeq 0.4 \mathrm{MPa})$. The nominal pressure is associated with a nominal volume of $21 \mathrm{ml}$.

In this paper, the balloon was modelled as a pressurised cylindrical thin-walled vessel with hemispherical ends as shown in Figure 3. The thin-walled vessel assumption is accurate for ratios of thickness to inside diameter less than $1 / 20$. Under these conditions, radial stress is negligible, and hoop and longitudinal stresses can be approximated as equally distributed across the wall thickness. Solving the equilibrium and assuming a linear elastic constitutive behaviour, the volumetric strains for thin-walled cylinders and spheres can be written as:

$$
\begin{aligned}
\epsilon_{c} & =\frac{\left(p-p_{e}\right) D}{4 t_{c} E}(5-4 \nu) \\
\epsilon_{s} & =\frac{3\left(p-p_{e}\right) D}{4 t_{s} E}(1-\nu)
\end{aligned}
$$

where $p$ is the intra-balloon absolute pressure, $p_{e}$ is the external pressure, $D$ is the unstretched diameter, $t_{c}$ the thickness of the cylinder, $t_{s}$ the thickness of the hemispherical portions, $E$ the Young's modulus and $\nu$ the Poisson's ratio. Considering constant external atmospheric pressure, Equations 1 and 2 can be combined to define a general analytical relation between the instantaneous balloon volume and the intra-balloon absolute pressure valid during free inflation:

$$
V(p)=\frac{\pi L D^{2}}{4}\left(1+\epsilon_{c}\right)+\frac{\pi D^{3}}{6}\left(1+\epsilon_{s}\right)+c
$$

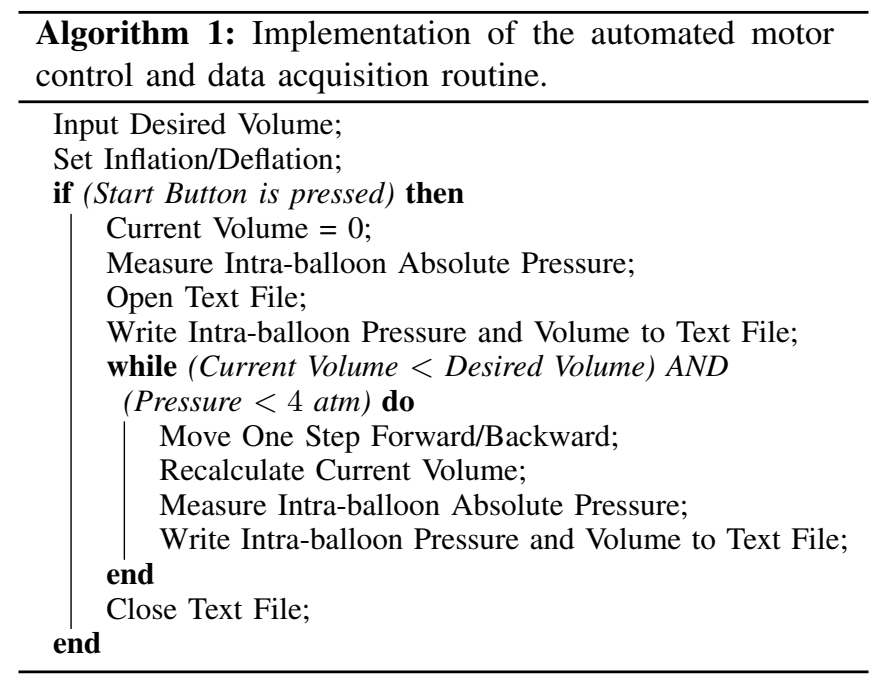

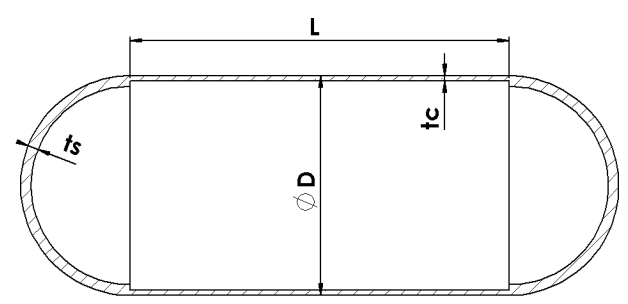

Fig. 3. The balloon catheter was modelled as a cylindrical thin-walled vessel with hemispherical ends.

In Equation 3, $L$ is the unstretched length of the cylindrical region and $c$ is a constant accounting for the presence of the guide wire pipe and potential residual saline solution inside the balloon cavity. To fully characterise the mathematical model, the parameters $D, L, E, \nu, t_{c}, t_{s}$ and $c$ need to be determined. The values for $E$ and $\nu, 556 \mathrm{MPa}$ and 0.45 respectively, were experimentally determined from tensile tests on samples extracted from the actual device.

Since the geometric specifications provided by the manufacturer refer to the balloon in its pressurised configuration when the material is stretched, the 3D unstretched non-collapsed geometry was reconstructed to obtain $D$ and $L$. The catheter was connected to an open tank by means of a 4-way stopcock valve. Water was filled into the container until a distance of approximately $2 \mathrm{~cm}$ between the free surface of the fluid and the cylindrical surface of the balloon was reached. In such circumstances, the intra-balloon transmural pressure is approximately $200-400 \mathrm{~Pa}$. The associated diametrical stretch is of the order of $10^{-3} \mathrm{~mm}$ which is negligible. The stopcock valve was then closed. The device and the valve were disconnected from the reservoir and positioned in a polariscope to take high-resolution pictures. The outer geometry of the balloon was reconstructed by importing the images into CAD software (Solidworks, Dassault Systèmes, Vélizy-Villacoublay, France). According to measurements on the reconstructed part, $D$ was set to $20.75 \mathrm{~mm}$ and $L$ to $35.82 \mathrm{~mm}$.

The thickness of the balloon $t_{c}$ was estimated from the analytical expression derived for the determination of the stretched diameter $D_{s}$ as the transmural pressure increases:

$$
D_{s}=D+\frac{\left(p-p_{e}\right) D^{2}}{4 t_{c} E}(2-\nu)
$$

By imposing a value of $D_{s}$ equal to $23 \mathrm{~mm}$ for a transmural pressure of $4 \mathrm{~atm}$, as per device specifications, Equation 4 can be solved for $t_{c}$ (in this case, $t_{c}=0.05 \mathrm{~mm}$ ). Experimental free inflation data were acquired to estimate the remaining unknowns $t_{s}$ and $c$. The balloon was inflated to $p=4 \mathrm{~atm}$, utilising the platform described in Section II-A. The test was repeated 5 times, and results were averaged to get a characteristic absolute pressure-volume $(p-v)$ curve for the balloon. The averaged $p-v$ curve was subsequently imported into Matlab (MathWorks, Natick, MA, US) and smoothened using a robust local regression (loess) filter. The critical point at which the balloon starts stretching was identified within the dataset as the maximum of the numerical second derivative of the pressure with respect to 

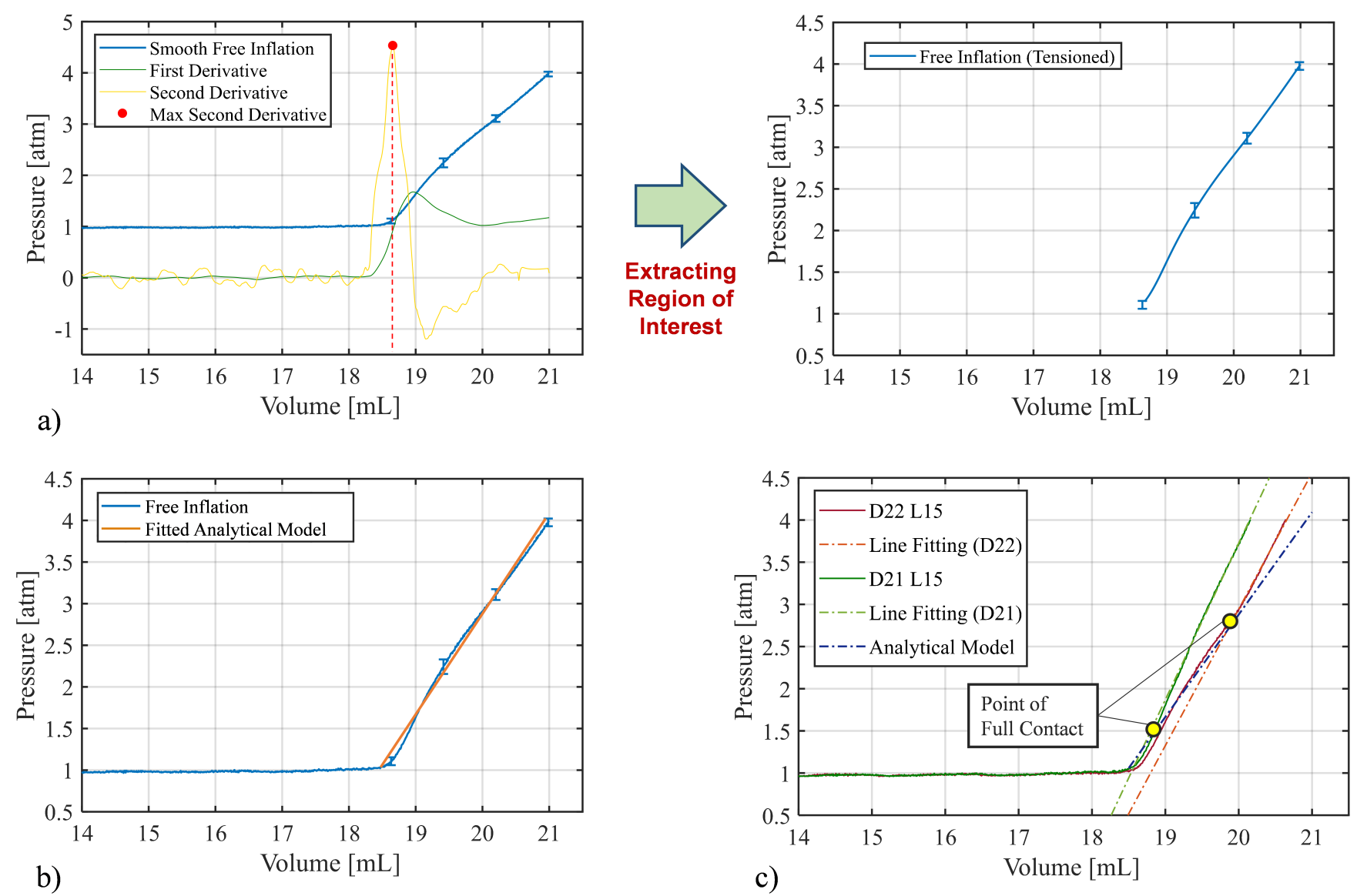

Fig. 4. a) Extracting the portion of the balloon characteristic free inflation curve where the material is tensioned b) Average experimental free inflation of the balloon catheter with standard deviation bars and fitted analytical model (absolute pressure-volume curve) c) Example of iterative linear regression performed on $p-v$ curves obtained from experimental valvuloplasty on idealised annuli with diameters of 22 and $21 \mathrm{~mm}$ (and $15 \mathrm{~mm}$ length). The point of full contact is where the $p-v$ curve deviates from the free inflation curve, as a result of the balloon-annulus contact.

the volume. The portion of the curve where the material is tensioned was extracted accordingly (Figure 4a). Finally, nonlinear least-squares data fitting was performed to fit the analytical expression of the volume (Equation 3) to the extracted dataset. Since $t_{s}$ and $c$ are the only degrees of freedom of the function $V(p)$, they were calculated as a result of the fitting. An optimal fit, characterised by a rootmean-square error (RMSE) equal to $55.59\left(R^{2}=0.99\right)$ was achieved for $t_{s}=0.05 \mathrm{~mm}$ and $c=1.66 \mathrm{ml}$ (Figure 4b).

\section{The sizing algorithm}

The proposed approach is built upon the assumption that the diameter of the balloon, measured at the time when full contact with the annular walls is first attained, equals the annular diameter. Therefore, sizing can be performed indirectly by identifying the point within the acquired dataset, at which the $p-v$ curve deviates from the free inflation curve, and estimating the corresponding balloon diameter. This is achieved by iteratively performing linear regression on the $p-v$ dataset (as shown in Figure $4 c$ and Algorithm 2). In each iteration, the data point with the lowest volume value is removed, a line is fitted to the updated dataset and the associated RMSE is extracted. The process stops when the
RMSE is smaller than an empirically set threshold (0.03). Thus, the equation of a line, which approximates the portion of the processed $p-v$ curve deviating from the free inflation curve, is obtained. The symbolic mathematical capabilities of Matlab can then be exploited to find the desired point as the intersection between the fitted line and the model function $V(p)$. The corresponding pressure can be substituted in Equation 4 to determine the balloon diameter.

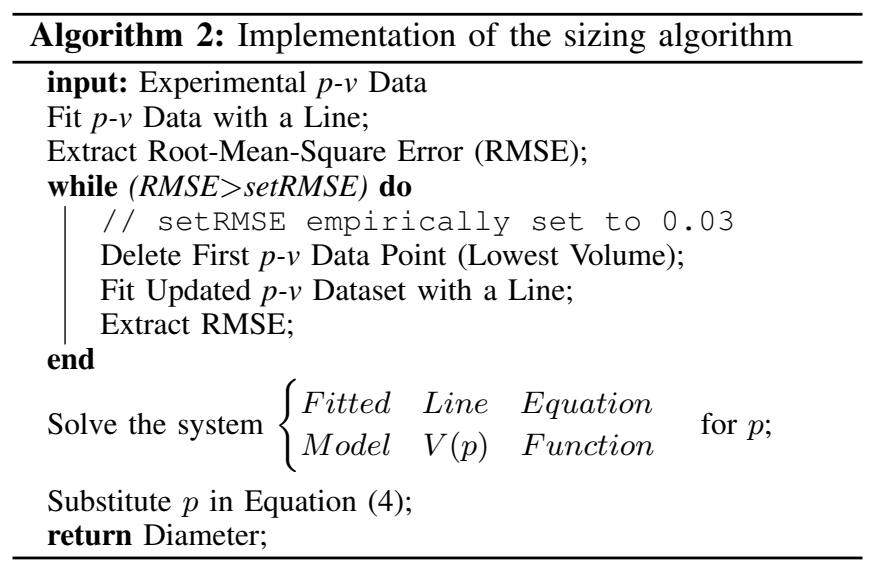




\section{EXPERIMENTAl SETUP, Results AND Discussion}

\section{A. Experimental setup and test protocol}

To validate the proposed methodology, the balloon catheter was inserted into aortic annular phantoms that were designed by approximating the implantation site as a rigid circular cylinder (Figure 5). This assumption is considered valid as in vivo studies have shown that the annular perimeter of patients affected by AS undergoes negligible deformation over the cardiac cycle [22], [26]. Also, after prosthetic valve implantation, significant changes in the annular diameter were not observed [27], [28].

Five different diameters were selected to take into account typical (21 and $22 \mathrm{~mm}$ ) and extremely aggressive (18, 19 and $20 \mathrm{~mm}$ ) balloon-annulus sizing ratios [5]. The idealised annuli were machined in four $5 \mathrm{~mm}$-thick acrylic plates specifying a diametral dimensional tolerance of $\pm 0.1 \mathrm{~mm}$. The plates could then be stacked to obtain the lengths of $15 \mathrm{~mm}$ and $20 \mathrm{~mm}$ delimiting reported annular length variability in adult human aortic valves [29].

The procedure adopted to perform the balloon inflation inside the annular phantoms was divided into the following steps:

1) The inflation device was filled with saline solution and the balloon was completely emptied.

2) The balloon was crimped and placed inside the phantom. No particular precautions were taken to enforce any axial positioning of the balloon.

3) $11 \mathrm{ml}$ of saline solution were injected into the balloon. This pre-inflation allowed to achieve the transition from non-stressed balloon material to stressed balloon material and desired intra-balloon pressure with only one additional stroke of the plunger.

4) The inflation device was refilled.

5) Water was pumped into the balloon, until the intraballoon absolute pressure reached 4 atm.

A flow rate of about $0.076 \frac{\mathrm{ml}}{\mathrm{s}}$ was set for all experimental tests. For each annular phantom, five inflations and deflations were performed. The acquired $p-v$ curves were then imported into Matlab and automatically processed by the sizing algorithm.

\section{B. Experimental results and discussion}

A unique $p-v$ curve, for each phantom configuration, was obtained by averaging the results of the five tests (as shown in Figure 6). It can be observed from the graph that, for annular diameters bigger than or equal to the balloon unstretched diameter $(\geq 20.75 \mathrm{~mm})$, the full contact with the annular wall is immediately identifiable from the point at which the $p-v$ curve deviates from the free inflation curve. The algorithm presented in Section II-C considers this observation. The coordinates of this point are independent from the length of the phantom. The annular length only affects the slope of the curve after full contact. More specifically, the higher the annular length, the steeper the portion of the curve that deviates from the free inflation curve is.

For annular diameters smaller than $20.75 \mathrm{~mm}$, the balloon reaches full contact with the annulus, while its wall is not

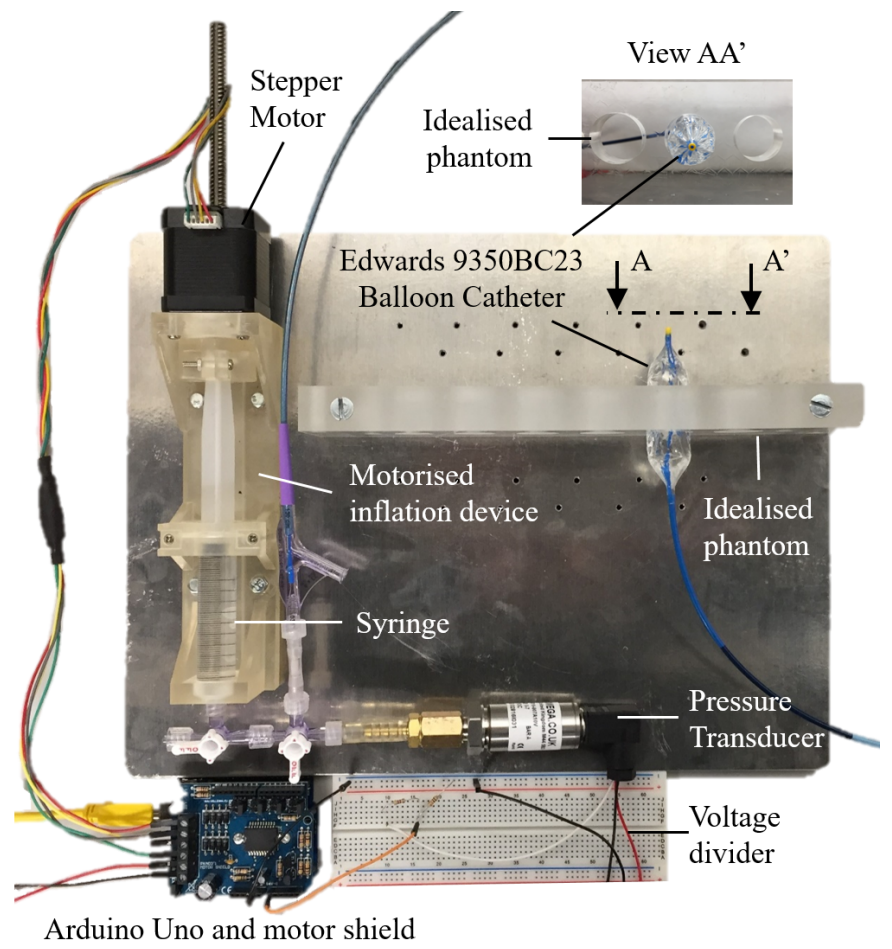

Fig. 5. Sizing idealised aortic annular phantoms with a robotised soft robotic balloon catheter: Top view of the experimental setup and front view of the balloon catheter inside the idealised phantom.

tensioned. Therefore, the departure from the free inflation curve is not directly associated with the annular diameter. Rather, it is caused by the balloon inability to deploy freely, which produces an anticipated tensioning of the balloon material. For these diameters, the point at which the balloon material starts tensioning depends significantly on the annular length. In these circumstances, it is impossible to obtain a direct and unbiased estimate of the annular diameter, from basic considerations on $p-v$ data. This observation supports our decision to develop a mathematical model that does not describe the non-tensioned phase of the balloon. On this basis, the sizing was only performed on phantoms with diameter larger than $20.75 \mathrm{~mm}$ (21 and $22 \mathrm{~mm}$ ). The

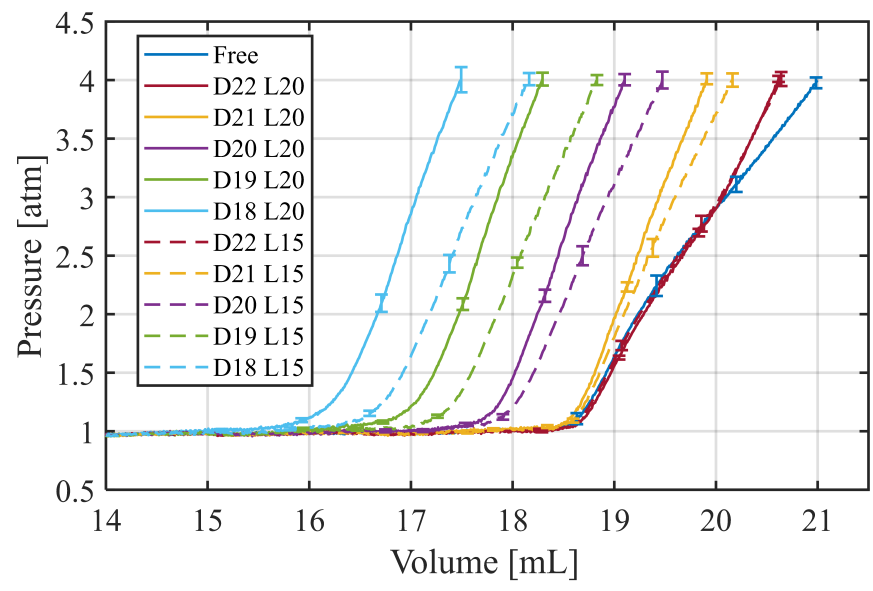

Fig. 6. Comparison between average absolute pressure-volume curves obtained for different diameters (D) and lengths (L). 
TABLE I

NOMINAL VS EXPERIMENTALLY DETERMINED ANNULAR DIAMETER

\begin{tabular}{ccccc}
\hline $\begin{array}{c}\text { Annular } \\
\text { Diameter } \\
{[\mathrm{mm}]}\end{array}$ & $\begin{array}{c}\text { Annular } \\
\text { Length } \\
{[\mathrm{mm}]}\end{array}$ & $\begin{array}{c}\text { Average } \\
\text { Measured Diameter } \\
{[\mathrm{mm}]}\end{array}$ & $\begin{array}{c}\text { Standard } \\
\text { Deviation } \\
{[\mathrm{mm}]}\end{array}$ & $\begin{array}{c}\text { Average } \\
\text { Error } \\
{[\%]}\end{array}$ \\
\hline 22 & 20 & 22.002 & \pm 0.03 & 0.01 \\
21 & 20 & 20.908 & \pm 0.05 & 0.44 \\
22 & 15 & 22.204 & \pm 0.11 & 0.93 \\
21 & 15 & 21.133 & \pm 0.10 & 0.63 \\
\hline
\end{tabular}

experimental results, summarised in Table I, clearly confirm that intra-balloon $p-v$ data can be used to determine the annular diameters with good precision. The average error is calculated as in Equation 5.

$$
\underset{\text { Error }}{\text { Average }}=\left|\frac{\text { Average Measured } \varnothing-\text { Annular } \varnothing}{\text { Annular } \varnothing}\right|
$$

In the examined cases, the proposed approach exhibits good repeatability (maximum standard deviation $\pm 0.11 \mathrm{~mm}$ ) and high accuracy (maximum average error $0.93 \%$ ).

The discrepancies in the average measurements obtained for the same diameter and different annular lengths are in agreement with the dimensional tolerances specified for the manufacturing of the phantoms, and the inherent statistical variability of the experimental data.

\section{CONCLUSIONS}

In this paper, we presented a new approach to determine the diameter of the aortic annulus exploiting intra-balloon pressure and volume data acquired from a robotic valvuloplasty balloon catheter. A robotic inflation device, capable of collecting real-time intra-balloon pressure and volume data, was designed and interfaced with a commercially available BAV balloon catheter. To develop a characterised analytical model of the balloon free inflation, the balloon was approximated as a pressurised cylindrical thin-walled vessel with hemispherical ends. A sizing algorithm that combines the mathematical model and an iterative linear regression method was implemented to estimate the annular diameter from the associated experimental $p-v$ curve. Our solution was tested on idealised aortic annular phantoms. Experimental results confirm that $p-v$ data can be used to determine annular diameters larger than the unstretched diameter of the balloon catheter with good repeatability and high precision - oversizing is current practice as TAVI operators choose a maximum $10 \%$ larger balloon compared to the annular diameter [5]. Therefore, a $23 \mathrm{~mm}$ BAV catheter, like the one used in this study, would not be employed for annular diameters smaller than $20.9 \mathrm{~mm}$, which is still in the identified measurable range. The described approach could be easily transferred to other standard balloon catheters of different sizes, already used in routine clinical practice. Therefore, it could be employed alongside traditional imaging-based sizing techniques to improve TAVI device selection, and is intended as a verification tool, which could potentially refine results obtained from imaging modalities, such as TEE $\backslash$ TTE (see Section I).

Though several studies in the literature demonstrated that the aortic annulus in patients affected by AS is a substantially stiff structure and our chosen idealised phantoms would be a reasonable approximation to represent severe stenosis, a variable degree of compliance could be added to the annular phantoms to reproduce milder forms of the disease. In future work, experimental tests on relatively compliant aortic annular phantoms with patient-specific geometries will be performed. In principle, the proposed approach would work on compliant phantoms as well, given that their stiffness produces a detectable deviation in the $p$ - $v$ curve from the free inflation curve. Furthermore, a strategy to assess the mechanical properties of the aortic annulus from intra-balloon pressure and volume data will be implemented. This will provide the operator with supplementary helpful information for TAVI device selection, as the mechanical response of the implantation region has a critical impact on the radial forces applied by prosthetic valves. Finally, we will investigate a mathematical model of the balloon which considers nonlinearities of the balloon material and large deformations.

\section{ACKNOWLEDGMENT}

This work is supported by the Engineering and Physical Sciences Research Council (grant numbers: EP/N509577/1 and EP/S014039/1) and by the Springboard Award of the Academy of Medical Sciences (grant number: SBF0031109).

\section{REFERENCES}

[1] Matthew J Czarny and Jon R Resar. Diagnosis and management of valvular aortic stenosis. Clinical Medicine Insights: Cardiology, 8:CMC-S15716, 2014.

[2] Martin B Leon, Craig R Smith, Michael Mack, D Craig Miller, Jeffrey W Moses, Lars G Svensson, E Murat Tuzcu, John G Webb, Gregory P Fontana, Raj R Makkar, et al. Transcatheter aorticvalve implantation for aortic stenosis in patients who cannot undergo surgery. New England Journal of Medicine, 363(17):1597-1607, 2010.

[3] Philip Adamson and Nicholas Cruden. Emergency interventions for the treatment of decompensated aortic stenosis. Heart, 104(1):4-5, 2018.

[4] Benedetta Biffi, Giorgia M Bosi, Valentina Lintas, Rod Jones, Spyros Tzamtzis, Gaetano Burriesci, Francesco Migliavacca, Andrew M Taylor, Silvia Schievano, and Giovanni Biglino. Numerical model of a valvuloplasty balloon: in vitro validation in a rapid-prototyped phantom. Biomedical Engineering online, 15(1):37, 2016.

[5] Thomas R Keeble, Arif Khokhar, Mohammed Majid Akhtar, Anthony Mathur, Roshan Weerackody, and Simon Kennon. Percutaneous balloon aortic valvuloplasty in the era of transcatheter aortic valve implantation: a narrative review. Open Heart, 3(2):e000421, 2016.

[6] Craig R Smith, Mack Michael J Leon, Martin B, D Craig Miller, Jeffrey W Moses, Lars G Svensson, E Murat Tuzcu, John G Webb, Gregory P Fontana, Raj R Makkar, et al. Transcatheter versus surgical aortic-valve replacement in high-risk patients. New England Journal of Medicine, 364(23):2187-2198, 2011.

[7] Alain Cribier, Helene Eltchaninoff, Assaf Bash, Nicolas Borenstein, Christophe Tron, Fabrice Bauer, Genevieve Derumeaux, Frederic Anselme, François Laborde, and Martin B Leon. Percutaneous transcatheter implantation of an aortic valve prosthesis for calcific aortic stenosis: first human case description. Circulation, 106(24):30063008,2002

[8] Martin B Leon, Craig R Smith, Michael J Mack, Raj R Makkar, Lars G Svensson, Susheel K Kodali, Vinod H Thourani, E Murat Tuzcu, D Craig Miller, Howard C Herrmann, et al. Transcatheter or surgical aortic-valve replacement in intermediate-risk patients. New England Journal of Medicine, 374(17):1609-1620, 2016. 
[9] Usaid K Allahwala, Peter S Hansen, Edward J Danson, Nicola Straiton, Ajay Sinhal, Darren L Walters, and Ravinay Bhindi. Transcatheter aortic valve implantation: current trends and future directions. Future Cardiology, 12(1):69-85, 2016.

[10] Helge Möllmann, Won-Keun Kim, Jörg Kempfert, Thomas Walther, and Christian Hamm. Complications of transcatheter aortic valve implantation: how to avoid and treat them. Heart, pages 900-908, 2015.

[11] Hans R Figulla, John G Webb, Alexander Lauten, and Ted Feldman. The transcatheter valve technology pipeline for treatment of adult valvular heart disease. European Heart Journal, 37(28):2226-2239, 2016.

[12] Andrew A Klein, Nikolas J Skubas, and Joerg Ender. Controversies and complications in the perioperative management of transcatheter aortic valve replacement. Anesthesia \& Analgesia, 119(4):784-798, 2014.

[13] Christian W Hamm, Mani Arsalan, and Michael J Mack. The future of transcatheter aortic valve implantation. European Heart Journal, 37(10):803-810, 2015.

[14] Susheel K Kodali, Mathew R Williams, Craig R Smith, Lars G Svensson, John G Webb, Raj R Makkar, Gregory P Fontana, Todd M Dewey, Vinod H Thourani, Augusto D Pichard, et al. Two-year outcomes after transcatheter or surgical aortic-valve replacement. New England Journal of Medicine, 366(18):1686-1695, 2012.

[15] Michael Scharfschwerdt, Roza Meyer-Saraei, Claudia Schmidtke, and Hans-Hinrich Sievers. Hemodynamics of the edwards sapien $\mathrm{xt}$ transcatheter heart valve in noncircular aortic annuli. The Journal of Thoracic and Cardiovascular Surgery, 148(1):126-132, 2014.

[16] Yoshio Maeno, Yigal Abramowitz, Sung-Han Yoon, Hasan Jilaihawi, Sharjeel Israr, Masaki Miyasaka, Hiroyuki Kawamori, Yoshio Kazuno, Tanya Rami, Nobuyuki Takahashi, et al. Transcatheter aortic valve replacement with different valve types in elliptic aortic annuli. Circulation Journal, 81(7):1036-1042, 2017

[17] Alfredo G Cerillo, Massimiliano Mariani, Sergio Berti, and Mattia Glauber. Sizing the aortic annulus. Annals of Cardiothoracic Surgery, 1(2):245, 2012.

[18] David Messika-Zeitoun, Jean-Michel Serfaty, Eric Brochet, Gregory Ducrocq, Laurent Lepage, Delphine Detaint, Fabien Hyafil, Dominique Himbert, Nicoletta Pasi, Jean-Pierre Laissy, et al. Multimodal assessment of the aortic annulus diameter: implications for transcatheter aortic valve implantation. Journal of the American College of Cardiology, 55(3):186-194, 2010.

[19] Kyoko Otani, Masaaki Takeuchi, Kyoko Kaku, Lissa Sugeng, Hidetoshi Yoshitani, Nobuhiko Haruki, Toshiyuki Ota, Victor MorAvi, Roberto M Lang, and Yutaka Otsuji. Assessment of the aortic root using real-time 3D transesophageal echocardiography. Circulation Journal, 74(12):2649-2657, 2010.

[20] Daniel Kretzschmar, Alexander Lauten, Bjoern Goebel, Torsten Doenst, Tudor C Poerner, Markus Ferrari, Hans R Figulla, and Ali Hamadanchi. Optimal prosthesis sizing in transcatheter aortic valve implantation by exclusive use of three-dimensional transoesophageal echocardiography. Clinical physiology and functional imaging, 36(2):99-105, 2016.

[21] Jörg Kempfert, Arnaud Van Linden, Lukas Lehmkuhl, Ardawan J Rastan, David Holzhey, Johannes Blumenstein, Friedrich W Mohr, and Thomas Walther. Aortic annulus sizing: echocardiographic versus computed tomography derived measurements in comparison with direct surgical sizing. European Journal of Cardio-Thoracic Surgery, 42(4):627-633, 2012.

[22] Darra T Murphy, Philipp Blanke, Shalan Alaamri, Christopher Naoum, Ronen Rubinshtein, Gregor Pache, Bruce Precious, Adam Berger, Rekha Raju, Danny Dvir, et al. Dynamism of the aortic annulus: effect of diastolic versus systolic CT annular measurements on device selection in transcatheter aortic valve replacement (tavr). Journal of Cardiovascular Computed Tomography, 10(1):37-43, 2016.

[23] Patrick W Serruys, Nicolo Piazza, Alain Cribier, John Webb, JeanClaude Laborde, and Peter de Jaegere. Transcatheter aortic valve implantation: tips and tricks to avoid failure. CRC Press, 2009.

[24] Vasilis C Babaliaros, Zahid Junagadhwalla, Stamatios Lerakis, Vinod Thourani, David Liff, Edward Chen, Thomas Vassiliades, Clay Chappell, Nathan Gross, Ateet Patel, et al. Use of balloon aortic valvuloplasty to size the aortic annulus before implantation of a balloon-expandable transcatheter heart valve. JACC: Cardiovascular Interventions, 3(1):114-118, 2010.

[25] Vasilis C Babaliaros, David Liff, Edward P Chen, Jason H Rogers,
Ryan A Brown, Vinod H Thourani, Robert A Guyton, Stamatios Lerakis, Arthur E Stillman, Paolo Raggi, et al. Can balloon aortic valvuloplasty help determine appropriate transcatheter aortic valve size? JACC: Cardiovascular Interventions, 1(5):580-586, 2008.

[26] Ashraf Hamdan, Victor Guetta, Eli Konen, Orly Goitein, Amit Segev, Ehud Raanani, Dan Spiegelstein, Ilan Hay, Elio Di Segni, Michael Eldar, et al. Deformation dynamics and mechanical properties of the aortic annulus by 4-dimensional computed tomography: insights into the functional anatomy of the aortic valve complex and implications for transcatheter aortic valve therapy. Journal of the American College of Cardiology, 59(2):119-127, 2012.

[27] Carl J Schultz, Annick Weustink, Nicolo Piazza, Amber Otten, Nico Mollet, Gabriel Krestin, Robert J van Geuns, Pim de Feyter, Patrick WJ Serruys, and Peter de Jaegere. Geometry and degree of apposition of the corevalve revalving system with multislice computed tomography after implantation in patients with aortic stenosis. Journal of the American College of Cardiology, 54(10):911-918, 2009.

[28] Philipp Blanke, Matthias Siepe, Jochen Reinöhl, Manfred Zehender, Friedhelm Beyersdorf, Christian Schlensak, Mathias Langer, and Gregor Pache. Assessment of aortic annulus dimensions for edwards sapien transapical heart valve implantation by computed tomography: calculating average diameter using a virtual ring method. European Journal of Cardio-Thoracic Surgery, 38(6):750-758, 2010.

[29] Mano J Thubrikar. The aortic valve. CRC press, 1989. 